\title{
Reconfigurable sticker label electronics manufactured from nanofibrillated cellulose- based self-adhesive organic electronic materials
}

\author{
Jun Kawahara, Peter Andersson Ersman, Xin Wang, Göran Gustafsson, Hjalmar Granberg \\ and Magnus Berggren
}

\section{Linköping University Post Print}

\section{Tweet}

N.B.: When citing this work, cite the original article.

Original Publication:

Jun Kawahara, Peter Andersson Ersman, Xin Wang, Göran Gustafsson, Hjalmar Granberg and Magnus Berggren, Reconfigurable sticker label electronics manufactured from nanofibrillated cellulose-based self-adhesive organic electronic materials, 2013, Organic electronics.

http://dx.doi.org/10.1016/j.orgel.2013.07.013

Copyright: Elsevier

http://www.elsevier.com/ 


\section{Reconfigurable sticker label electronics manufactured from nanofibrillated cellulose-based self-adhesive organic electronic materials}

\section{By Jun Kawahara, Peter Andersson Ersman*, Xin Wang, Göran Gustafsson, Hjalmar Granberg and Magnus Berggren}

[*] Dr. P. Andersson Ersman (Corresponding Author), Dr. X. Wang, Dr. G. Gustafsson and J. Kawahara

Printed Electronics, Acreo Swedish ICT AB

Bredgatan 34, SE-60221, Norrköping, Sweden

E-mail: peter.andersson.ersman@acreo.se

Prof. M. Berggren, J. Kawahara

Department of Science and Technology, Linköping University

Bredgatan 33, SE-60174, Norrköping, Sweden

J. Kawahara

R\&D Strategy Department, Lintec Corporation

5-14-42 Nishiki-cho, Warabi, Saitama, 3350005, Japan

Dr. H. Granberg

Innventia $\mathrm{AB}$

Drottning Kristinas väg 61, SE-11428 Stockholm, Sweden

Keywords: Cellulose nanofiber, electrochromic display, electrochemical transistor, selfadhesion, electronic system integration

Abstract: Low voltage operated electrochemical devices, such as electrochromic displays, electrochemical transistors and electrolyte capacitors can be produced from electrically conducting polymers and polyelectrolytes. Here, we report how such polymers and polyelectrolytes can be casted together with nanofibrillated cellulose (NFC) derived from wood. The resulting films, which carry ionic or electronic functionalities, are all-organic, disposable, light-weight, flexible, self-adhesive, elastic and self-supporting. The mechanical and self-adhesive properties of the films enable us to achieve simple and flexible electronic systems by assembling the films into various kinds of components using a "cut and stick" method. Additionally, the self-adhesive surfaces provide us with a new concept that not only allows for simplified system integration of printed electronic components, but also allows for a unique possibility to detach and reconfigure one or several subcomponents by a "peel and stick" method to create yet another device configuration. This is demonstrated by a stack of two films that first served as the electrolyte layer and the pixel electrode of an electrochromic 
display, which then was detached from each other and transferred to another configuration, thus becoming the electrolyte and gate electrode of an electrochemical transistor. Further, smart pixels, consisting of the combination of one electrochromic pixel and one electrochemical transistor, have successfully been manufactured with the NFC-hybridized materials. The concept of system reconfiguration was further explored by that a pixel electrode charged to its colored state could be detached and then integrated on top of a transistor channel. This resulted in spontaneous discharging and associated current modulation of the transistor channel without applying any additional gate voltage. Our peel and stick approach promises for novel reconfigurable electronic devices, e.g. in sensor, label and security applications.

\section{Introduction}

Since its discovery about 2000 years ago, papers are one of the most commonly manufactured and utilized sheet materials in our world; it represents the planar carrier that we utilize to record, transfer and share printed information. In fact, it is the largest surface ever manufactured by mankind. During the digital revolution, paper has certainly been challenged. However, nowadays, rather thanks to the digital revolution the interest for paper as the carrier for information is regained. One of the prime reasons for this revival is the birth of the technology and science field known as printed electronics. From an application point of view paper now turns into the carrier for flexible electronics, systems that can be manufactured using the same printing tools that were originally developed for graphics and texts. Printed electronics defined on paper labels, package board and on fine paper, is expected to extend the function of paper and to make paper products connected to the digital world.

Paper is based on wood fibers comprising the most abundant organic compound derived from biomass, namely cellulose. One such renewable cellulose material, nanofibrillated cellulose (NFC), has attracted a very large interest during the last decade. This 
material emanates from the cellulose fibrils inside the wood fibre wall and the fibrils are liberated in different ways using both chemical treatment and high-pressure homogenization to separate the fibrils from each other and to stabilize them in aqueous dispersion. ${ }^{[1]}$ The attraction of NFC stems from their interesting intrinsic properties such as its high specific surface area, flexibility, high aspect ratio of the fibrils, good mechanical properties, and its film forming capacity. NFC dispersions converted into "nanopaper" films can be transparent, reach mechanical properties similar to that of cast iron, ${ }^{[2]}$ or show very good oxygen barrier properties. NFC has also been used as a filler to provide reinforcement, flexibility and transparency in nanocomposites and displays. ${ }^{[3]}$

The field of printed electronics is presently attracting a lot of interest since it promises for distributed intelligence and monitoring; features that are expected to combat some of the challenges in our society related to health care, monitoring, logistics and safety. A tremendous variety of components have been developed and manufactured on flexible substrates using different kinds of coating, printing and lamination tools. ${ }^{[4]}$ Examples include batteries, capacitors, solar cells, displays, diodes, transistors and logic circuits. To achieve true printed electronics applications, typically several different kinds of components need to be integrated into a monolithic flexible system. However, integration of printed electronic subcomponents has proven to be a major challenge with respect to the merger and compatibility of different printing technologies, materials and post-processing steps. In fact, in many cases specific manufacturing steps are mutually exclusive since some steps tend to destroy or deteriorate features and components manufactured earlier in the integration scheme. This then implies a decreased production yield that can only be solved by typically increasing the cost for production and integration. In addition to this, the complexity of such production process also brings along the requirement of huge investments of non-standard manufacturing equipment. The overall mindset when developing the printed electronics platform must therefore aim at minimizing the number of materials and process steps, as well as trying to 
make the processes of different subcomponents compatible with each other by utilizing the same kind of materials for different functionalities. ${ }^{[5]}$

One of the major advantages of using electrochemical devices, based on for instance an electrochemically active conjugated polymer and an electrolyte, is that the very same material can be used for a versatility of device functions. The very same electrolyte component can be used both as the gate insulator in electrochemical and electrolyte-gated field effect transistors, and also as the electrolyte in electrochromic (EC) display devices, batteries and capacitors. PEDOT:PSS, poly(3,4-ethylenedioxythiophene) doped with poly(styrene sulfonate acid), can serve as the conductor, display electrode and also as the transistor channel in printed integrated circuits, such as displays, indicators and sensor systems. The PEDOT:PSS material is electrically conducting and optically transparent in its pristine state and it exhibits electrochromic switch characteristics and control of the conductivity upon electrochemical switching. PEDOT is a $\pi$-conjugated electronic polymer and the PSS phase serves as its counter ion. ${ }^{[6]}$ In the neutral state PEDOT appears deep blue ${ }^{[7]}$ and exhibit semiconducting properties. PEDOT can reversibly be switched in between its oxidized and neutral state. The electrochromic effect can be utilized in transmissive displays by using transparent electrolytes, or in a reflective mode of display operation in which the counter electrode is hidden under an opaque electrolyte. ${ }^{[8]}$ The latter version is typically used when PEDOT:PSS serves as both the counter and the pixel electrodes since such configuration maximizes the color switch contrast of the resulting display. However, there are different strategies in order to enhance the color switch contrast also for applications where transmission mode is desirable, for example by using a bottom display electrode and a top display electrode that together express a complementary EC switching characteristics with respect to each other. Polyaniline (PANI) is such an EC material that electrochemically switch color in a complementary fashion with respect to PEDOT, i.e. it exhibits a faint yellow, close to transparent, color in its reduced leucoemeraldine state, while PANI becomes dark blue, 
almost violet, in its oxidized pernigraniline state. Hence, an electrochromic display comprising a transparent electrolyte layer sandwiched by one PEDOT:PSS electrode and one PANI electrode can switch between a close to transparent pixel state to a dark black-blue colored state, where the latter color state is obtained by applying the positive and negative voltage to the PANI and PEDOT:PSS electrode, respectively. ${ }^{[9]}$

Besides reducing the number of different materials in printed electronics to achieve a resulting robust and rational platform for flexible electronics, one should also consider radically new integration concepts. In packaging and graphic art industry labels are commonly adhered to paper surfaces and products to generate a final integrated system or to extend the functionality of a specific product. Often, these add-on stickers include a coating that provides pressure-sensitive adhesion of the label to the surface of the carrier. In some cases those stickers can later be removed and then transferred to a different new carrier or item to serve yet another application.

Previous attempts on using "peel and stick" or "cut and stick" techniques have resulted in flexible thin film solar cells ${ }^{[10]}$ and free-standing dielectric layers for use in transistors, $^{[11]}$ respectively. However, the approach of the present study aims at functionalizing the carrier substrate in order to reduce the number of materials, and thus the number of processing steps, and also to develop a new integration concept for a reconfigurable electronic system by using self-adhesive electronic materials to enable a "cut, stick and peel” technology for printed electronics, i.e. to establish an electronic equivalence to the "scrapbook" or "sticker book".

\section{System reconfiguration of electronic devices}

\subsection{General description of the system reconfiguration process}

A semi-dried membrane of NFC mixed with appropriate plasticizers exhibits gel-like behavior when it comes to appearance and physical hardness. This property is partially 
remained in the fully dried state and hence a self-supporting film can be obtained thanks to the strong scaffold functionality of NFC, which originates from hydrogen bonds and/or ionic bonds inside the polymer matrix (not investigated further in this report), see Fig. 1. The resulting self-supporting composite films containing EC or electrolyte materials can weakly adhere to other solid functional substrate surfaces by simple lamination. Thanks to its weak adhering property the film can also be detached (here called “delaminated”) from a substrate while maintaining shape and functionality. This finally results in an extremely high degree of freedom to construct, disassemble and reconstruct multilayered printed electronic components. For example, in this report, NFC membranes hybridized with PEDOT:PSS (labeled as P:PSSNFC) are stacked onto NFC membranes hybridized with polyelectrolyte and white $\mathrm{TiO}_{2}$ pigment (denoted opaque Plyte-NFC) by hand, and a vertical symmetric electrochromic display (ECD) is established by that another layer of P:PSS-NFC is laminated on the other side of the Plyte-NFC.

Additionally, two other display architectures can also be prepared by simple lamination. A lateral symmetric ECD is constructed by the lamination of two separated P:PSS-NFC on the same side of the Plyte-NFC, where the two P:PSS-NFC are physically isolated. A vertical non-symmetric ECD is instead made from a drop-cast PANI layer on top of a Plyte-NFC (Plyte-NFC-PANI) and lamination of P:PSS-NFC on the opposite surface of the Plyte-NFC layer. These three ECD structures are drawn in Fig. 2 (a) to (c).

Upon delamination of the vertical symmetric ECD, one of the P:PSS-NFC layers is delaminated and removed from the other two layers, and the remaining bilayer can instead be laminated onto another functional surface in order to serve as e.g. the electrolyte and the ECD pixel electrode or the electrolyte and the gate electrode of an electrochemical transistor (ECT). This whole process, consisting of lamination, delamination and re-lamination, is here denoted “system reconfiguration”. Additionally, the NFC-based functional films typically are very 
robust and can be cut by scissors, folded or bended, hence, they have an appearance similar to the sticky notes used in scrapbooks or sticker books.

\subsubsection{Reconfiguration from ECD to ECT}

After characterizing the color switch contrast of the ECDs shown in Fig. 2 (a) to (c), the P:PSS-NFC layer on one side of the vertical symmetric ECD (Fig. 2 (b)) was delaminated and the remaining bilayer was cut in two pieces by using a pair of scissors. One of the cut pieces was manually laminated onto a PEDOT:PSS-based ECT channel prepared from an Orgacon EL-350 film patterned by a knife plotter tool. Here the laminated bilayer served as the gate electrode and the electrolyte layer of an ECT, and the patterned PEDOT:PSS on top of the PET substrate served as the drain, source and channel material, see Fig. 2 (e). The other cut piece was put onto another Orgacon foil such that color switching could be observed in order to prove the reconfiguration concept (data not recorded), see Fig. 2 (f).

\subsubsection{ECT characterization}

The structure of the resulting ECT is drawn in Fig. 3, although the schematic illustration shows a complete electrochromic smart pixel device that will be discussed later. Instead of completing the EC smart pixel device by preparing the top electrode of the ECD moiety, the ECT is obtained by connecting a DC power supply to the drain electrode such that the I-V curves of the ECT could be recorded separately.

\subsubsection{Reconfiguration from ECT to EC smart pixel}

The ECT characterized in 2.1.2 was further disassembled by delamination of the gate electrode bilayer. In combination with another piece of bilayer, which is mentioned in the end of 2.1.1 above, the two films were laminated onto another similarly patterned Orgacon film in order to create an EC smart pixel device, see Fig. 2 (g) and Fig. 3. In this report an EC smart 
pixel is defined as an ECD connected in series with an ECT, hence, the ECD coloration can be controlled by the conduction state of the addressing ECT. Here, upon reconfiguration, one of the laminated bilayers served as the gate electrode and the other was used as the pixel top electrode.

\subsubsection{EC smart pixel characterization}

The same external circuitry was connected as in the case of ECT characterization, except for that the pixel top electrode, and not the drain electrode, was connected to the equipment. The EC smart pixel characterization method has been reported previously ${ }^{[8]}$.

\subsubsection{Reconfiguration and duplication of the EC smart pixel}

The two bilayers of the EC smart pixel device described in 2.1.3 serve as the gate and the ECD pixel electrodes, and after delamination they were laminated onto another Orgacon film having the same pattern in order to create the same EC smart pixel architecture as in 2.1.3, which was characterized according to 2.1.4.

\subsubsection{Reconfiguration of a pre-charged hybridized NFC-based layer}

A vertical symmetric ECD was prepared according to Fig. 2 (b), followed by applying a voltage across the ECD, which in turn results in electrode charging. After delamination of the ECD, one P:PSS-NFC layer is fully oxidized while the other P:PSS-NFC layer is in its fully reduced state. The bilayer including reduced PEDOT:PSS was then reconfigured and transferred onto a PEDOT:PSS-based ECT channel such that the bilayer instead became the electrolyte and the gate electrode of an ECT. However, in this case the gate electrode is not connected to any power supply; it is only wired directly to the source electrode. So, since the gate electrode was electrochemically reduced in advance, it is expected that this charge will 
be equilibrated between the gate and the ECT channel, hence, current modulation of the ECT channel should occur just upon bringing the sheets into contact with each other.

\section{Device characteristics}

\subsection{Color switch contrast of the ECD}

EC pixels with three different architectures (Fig. 2 (a), (b) and (c)) were evaluated and the data is shown in Table 1. The measurement is performed by obtaining the CIE color space values $L^{*}, a^{*}$ and $b^{*}$ of the pixel electrode. Finally the color contrast value $\Delta \mathrm{E}^{*}$ is calculated from the following equation: ${ }^{[12]}$

$$
\Delta E^{*}=\sqrt{\left(L_{2}^{*}-L_{1}^{*}\right)^{2}+\left(a_{2}^{*}-a_{1}^{*}\right)^{2}+\left(b_{2}^{*}-b_{1}^{*}\right)^{2}}
$$

Here the subscripts " 1 ” and "2" for each color space parameter indicate the decolored (oxidized light blue) and colored (reduced deep blue) states of the PEDOT pixel electrode, i.e. the on and off states, respectively. The highest color contrast was obtained in a lateral pixel device; $\Delta \mathrm{E}^{*}=35.2$. This is somewhat lower as compared to recently reported PEDOT:PSSbased EC pixel devices. ${ }^{[13]}$ However, the obtained color contrast is considered to be sufficient for most display applications. The color contrast results and the appearances of the characterized displays are shown in Table 1 and Fig. 4.

\subsection{ECT characteristics}

The current-voltage (I-V) output curve for the depletion mode ECT device (Fig. 2 (e)) is shown in Fig. 5. Here the electric current through the channel between the drain and source electrodes $\left(\mathrm{I}_{\mathrm{DS}}\right)$ was recorded by sweeping the drain-source voltage $\left(\mathrm{V}_{\mathrm{DS}}\right)$ from 0 to $-1.0 \mathrm{~V}$ at an incremental step of $0.01 \mathrm{~V}$ for 6 different gate voltages $\left(\mathrm{V}_{\mathrm{G}}\right) ; 0 \mathrm{~V}$ at the first cycle and then increased in steps of $0.25 \mathrm{~V}$ until the last sweep at $1.25 \mathrm{~V}$. The time between two adjacent data points was $10 \mathrm{~ms}$. An on/off-ratio of approximately 100 was observed and the off-current level was about 60-80 nA. Both these values, especially the low off-current, are sufficient for 
the operation of an active matrix addressed display, see next section. The ECT device did not show any issues regarding delamination or degradation upon storage in ambient atmosphere (22 ${ }^{\circ} \mathrm{C}$ and $40 \% \mathrm{RH}$ ) for one month. Fig. 6 shows an ECT measured 19 and 31 days after device assembly. The relatively constant on- and off-current levels of the ECT demonstrate high degree of stability over extended periods of time.

\subsection{EC smart pixel: integration of ECD and ECT}

The function of the EC smart pixel, which herein is defined as the circuit integration of one ECT and one ECD, is validated by using two previously reported measurement methods. ${ }^{[8]}$ The two methods are chosen in order to investigate the two-folded functionality of the ECT in the smart pixel configuration; prevention of cross-talk along the addressing lines of the ECD devices in an active-matrix display and to improve the retention time of an ECD that has been updated to its colored state. The former is evaluated by monitoring that the pixel does not switch from its transparent off-state to its colored on-state upon applying $\mathrm{V}_{\mathrm{DS}}$ and $\mathrm{V}_{\mathrm{G}}$ (the ECT is in its off-state) simultaneously. The latter feature can be evaluated by applying $\mathrm{V}_{\mathrm{G}}$ after switching the ECD to its on-state, followed by turning off $\mathrm{V}_{\mathrm{DS}}$, which results in color retention of the ECD introduced by the non-conducting off-state of the ECT channel. Fig. 7 and Fig. 8 show the results of the two ECT-ECD smart pixel functionalities.

Fig. 7 indicates that the ECD is switched on only when $V_{D S}$ is turned on and when $V_{G}$ is $0 \mathrm{~V}$ (the ECT is in its on-state), as evidenced by the low leakage current flow through the EC smart pixel while the ECT channel is non-conducting. This is a required feature in order to prevent cross-talk effects along the EC pixel addressing lines in an active matrix addressed EC display; only one ECT per EC pixel addressing line is allowed to be in its conducting state. Fig. 8 demonstrates that the conduction state can control the retention time of the EC pixel. This has a direct impact on the power consumption in large active-matrix display systems. EC pixels are semi-bistable to their nature, thanks to their electrochemical and impedance 
characteristics. Further, the ability to control the color state of each EC pixel in a larger system is advantageous; not only between the on- and off-states but also to enable gray-scale levels. Here, the negative current peaks in Fig. 7 and Fig. 8 correspond to electrochemical reduction, i.e. switching to the non-conducting state and deep-blue coloration, of the PEDOT:PSS-based ECT channel or EC pixel electrode, while the positive current peak instead represents oxidation into the conducting and transparent form of the PEDOT:PSS in the ECT channel or EC pixel electrode. The area dependence on the switching time can be observed in the graphs; the relatively narrow current peaks correspond to the redox reactions of the ECT channel and the relatively broader current peaks represent color updates in the EC pixel. The switching time of the EC pixel is typically prolonged by high strain on the ECT channel, i.e. by simultaneously applying $\mathrm{V}_{\mathrm{DS}}$ and $\mathrm{V}_{\mathrm{G}}$ results in a reduction front propagating within the PEDOT:PSS channel outside the electrolyte edge. This is reflected in the broadened coloration current peak starting at $\mathrm{t} \sim 45 \mathrm{~s}$ in Fig. 7. There are several available routes how to circumvent the issue with long switching times in ECTs and EC smart pixels, this is however not the focus of the present work. ${ }^{[14]}$

By combining the measurements and results given in Fig. 7 and Fig. 8 we draw the conclusion that the color state of the EC pixel can be properly controlled by the applied gate voltage to the ECT device, and practically no leakage current is observed.

\subsection{Reconfiguration test of NFC-hybridized electrochemical devices}

Various kinds of electronic devices have been established by combining nanofibrillated cellulose (NFC) with either a polyelectrolyte or a conducting polymer, e.g. electrochromic displays, electrochemical transistors, electrochromic smart pixels and even electrolyte capacitors; components that together as integrated systems can form larger electronic systems, such as active matrix addressed displays, indicators, sensors and more. The devices obtained here utilize NFC in order to create ionically and electronically 
conducting films that are self-supporting, i.e. functionalized substrates. In addition to this, it is also possible to integrate, disintegrate and reconfigure the initially created subcomponents by using a "cut, stick and peel” technique similar to what is being used in a sticker book.

Reconfiguration of the gate electrode and the pixel electrode in the respective ECT and EC smart pixel devices, which resulted in a second set of devices, showed mostly identical performance as compared to the first set of devices shown in Fig. 5, 7 and 8 (data not shown). This demonstrates the robustness of the interfaces that are established upon reconfiguration of the functionalized substrates.

The system integration concept is also demonstrated by the measurement shown in Fig. 9. An electrochemical capacitor is formed by using a layer of Plyte-NFC as the intermediate layer, Orgacon as one of the electrodes and a layer of PEDOT:PSS-NFC as the other electrode. The latter electrode is then electrochemically reduced by applying a voltage of $\sim 5 \mathrm{~V}$ for $\sim 10$ seconds. The capacitor structure was then peeled apart by separating the bilayer from the Orgacon sheet, and the remaining bilayer was instead reconfigured to become the electrolyte and the gate electrode of an ECT by simply sticking it onto a $0.4 \times 3 \mathrm{~mm}^{2}$ PEDOT:PSS-based (Orgacon) channel. Prior to the attachment of the pre-charged gate electrode and its corresponding electrolyte, an on-current exceeding $50 \mu \mathrm{A}$ was flowing between the source and drain electrodes $\left(\mathrm{V}_{\mathrm{DS}}=-1 \mathrm{~V}\right)$. A spontaneous discharging of the gate electrode occurred, immediately after lamination, which then resulted in current modulation of the depletion mode ECT channel. Hence, the energy stored in the pre-charged conducting polymer was sufficient to alter the conduction state of the ECT device. The results demonstrate that organic electronic systems can be integrated, disintegrated and reconfigured into various flexible autonomous electronic systems by simple means and that information can be transferred between different devices.

\section{Conclusions}


A new concept for system integration of subcomponents for flexible electronics is demonstrated. The "cut, stick and peel” technique increases the degree of freedom to attach, detach and reconfigure electronic subcomponents located on a common substrate, making it a novel method for manufacturing of a variety of printed electronic systems that minimizes the number of materials and processing steps. The system integration technique is obtained by casting NFC with an electrochromic material or an electrolyte phase into a self-supporting film, which brings either electronic or ionic functionality into the film. Electrochemical devices are then easily created by stacking the self-adhesive films on top of each other. An electrochromic display pixel without ordinary plastic substrate (e.g. PET film), an electrochemical transistor where the NFC hybrid layers stick to the solid PEDOT:PSS channel and an electrochromic smart pixel composed of one pixel and one transistor were all successfully achieved. The components functioned well after performing the peel and stick reconfiguration of the NFC layers, and they also showed very good stability with respect to storage time in ambient atmosphere. The unique ability to integrate and reconfigure electronic systems based on self-supporting subcomponents will pave the way for the creation of more advanced flexible electronic circuits.

\section{Experimental}

Materials and equipment

Aqueous dispersion of anionic carboxylated NFC (0.5 wt\%, pH=7) was prepared at Innventia AB, Sweden, according to the method described by Wågberg et al.[15]. Aqueous dispersion of PEDOT:PSS “Baytron P HC”, toluene solution of polyaniline "PANIPOL T”, glycerol, and aqueous solution of poly(diallyl dimethyl ammonium chloride) (PDADMAC, polyelectrolyte with average M.W. $<100,000$ ), were purchased from Heraeus, Panipol Oy, and Sigma-Aldrich (the last two materials), respectively, and used without further treatments. 
PEDOT:PSS coated PET film “Orgacon EL-350” was purchased from AGFA. TiO2 powder “Kronos 2300” was purchased from Kronos and used as opacifier in an electrolyte.

Spectrophotometer (Datacolor Mercury, aperture diameter of $6.5 \mathrm{~mm}$ for the illumination, aperture diameter of $2.5 \mathrm{~mm}$ for the measurement, SCE (specular component excluded) mode and D65/10 degrees illumination) was used to measure the color contrast based on the CIE L*a*b* color coordinates. Keithley SourceMeter 2400 and HP/Agilent 4155B Parameter Analyzer were used to apply DC voltages and evaluate current vs. voltage characteristics of the devices.

Device preparation

P:PSS-NFC and Plyte-NFC (transparent and opaque) were prepared by mixing the following formulations described in dry wt\%: P:PSS-NFC <PEDOT:PSS / glycerol / NFC = 13.3 / 63.5 / 23.2>, transparent Plyte-NFC <PDADMAC / glycerol / NFC = 94.6 / 4.0 / 1.4>, opaque Plyte-NFC < PDADMAC / glycerol / TiO2 / NFC = 30.8 / 5.9 / 62.4 / 0.9>. Solutions of the different materials were mixed and dispersed using a Dispermill blender from ATP Engineering, about $5 \mathrm{ml}$ of each mixture was then poured into plastic petri dishes with a diameter of $50 \mathrm{~mm}$ and finally dried in ambient condition for two days. On one of the PlyteNFC, toluene-diluted PANI was drop cast and dried on one face (Plyte-NFC-PANI). Three different ECD structures were created by simple lamination of these hybridized films. The color switch contrast of each ECD was characterized by the spectrophotometer after connecting both electrodes to a DC power supply.

\section{Acknowledgements}

This work was supported by the Knut and Alice Wallenberg Foundation (Power Papers).

Received: ((will be filled in by the editorial staff))

Revised: ((will be filled in by the editorial staff)) Published online: ((will be filled in by the editorial staff)) 


\section{References}

[1] a) D. Klemm, F. Kramer, S. Moritz, T. Lindström, M. Ankerfors, D. Gray, A. Dorris, Angewandte Chemie International Edition 2011, 50, 5438; b) S. J. Eichhorn, A. Dufresne, M. Aranguren, N. E. Marcovich, J. R. Capadona, S. J. Rowan, C. Weder, W. Thielemans, M. Roman, S. Renneckar, W. Gindl, S. Veigel, J. Keckes, H. Yano, K. Abe, M. Nogi, A. N. Nakagaito, A. Mangalam, J. Simonsen, A. S. Benight, A. Bismarck, L. A. Berglund, T. Peijs, J Mater Sci 2010, 45, 1.

[2] M. Henriksson, L. A. Berglund, P. Isaksson, T. Lindström, T. Nishino, Biomacromolecules 2008, 9, 1579.

[3] a) S. C. M. Fernandes, C. S. R. Freire, A. J. D. Silvestre, C. Pascoal Neto, A. Gandini, L. A. Berglund, L. Salmén, Carbohydrate Polymers 2010, 81, 394; b) Y. Okahisa, A. Yoshida, S. Miyaguchi, H. Yano, Composites Science and Technology 2009, 69, 1958.

[4] a) M. Hočevar, U. O. Krašovec, M. Bokalič, M. Topič, W. Veurman, H. Brandt, A. Hinsch, Journal of Industrial and Engineering Chemistry 2013, Article in Press; b) H.-Y. Tseng, V. Subramanian, Org. Electron. 2011, 12, 249; c) R. Søndergaard, M. Hösel, D. Angmo, T. T. Larsen-Olsen, F. C. Krebs, Materials Today 2012, 15, 36; d) J. Noh, M. Jung, K. Jung, G. Lee, S. Lim, D. Kim, S. Kim, J. M. Tour, G. Cho, Org. Electron. 2011, 12, 2185; e) N. Zhao, M. Chiesa, H. Sirringhaus, Y. Li, Y. Wu, B. Ong, Journal of Applied Physics 2007, 101, p. 064513.

[5] M. Berggren, D. Nilsson, N. D. Robinson, Nat. Mater. 2007, 6, 3.

[6] F. Louwet, L. Groenendaal, J. Dhaen, J. Manca, J. Van Luppen, E. Verdonck, L. Leenders, Synthetic metals 2003, 135-136, 115.

[7] L. Groenendaal, F. Jonas, D. Freitag, H. Pielartzik, J. R. Reynolds, Adv. Mater. 2000, 12, 481. 
[8] J. Kawahara, P. Andersson Ersman, D. Nilsson, K. Katoh, Y. Nakata, M. Sandberg, M. Nilsson, G. Gustafsson, M. Berggren, Journal of Polymer Science Part B: Polymer Physics 2012, 51, 265.

[9] P. Andersson, R. Forchheimer, P. Tehrani, M. Berggren, Adv. Funct. Mater. 2007, 17, 3074.

[10] C. H. Lee, D. R. Kim, I. S. Cho, N. William, Q. Wang, X. Zheng, Sci. Rep. 2012, 2.

[11] K. H. Lee, M. S. Kang, S. Zhang, Y. Gu, T. P. Lodge, C. D. Frisbie, Adv. Mater. 2012, 24, 4457.

[12] a) R. J. Mortimer, K. R. Graham, C. R. G. Grenier, J. R. Reynolds, ACS Applied Materials \& Interfaces 2009, 1, 2269; b) T. Yijie, C. Haifeng, Z. Zhaoyang, X. Xiaoqian, Z. Yongjiang, Journal of Electroanalytical Chemistry 2013, 689, 142.

[13] J. Kawahara, P. Andersson Ersman, I. Engquist, M. Berggren, Org. Electron. 2012, 13, 469.

[14] P. Andersson Ersman, D. Nilsson, J. Kawahara, G. Gustafsson, M. Berggren, Org. Electron. 2013, 14, 1276.

[15] L. Wågberg, G. Decher, M. Norgren, T. Lindström, M. Ankerfors, K. Axnäs, Langmuir 2008, 24, 784. 


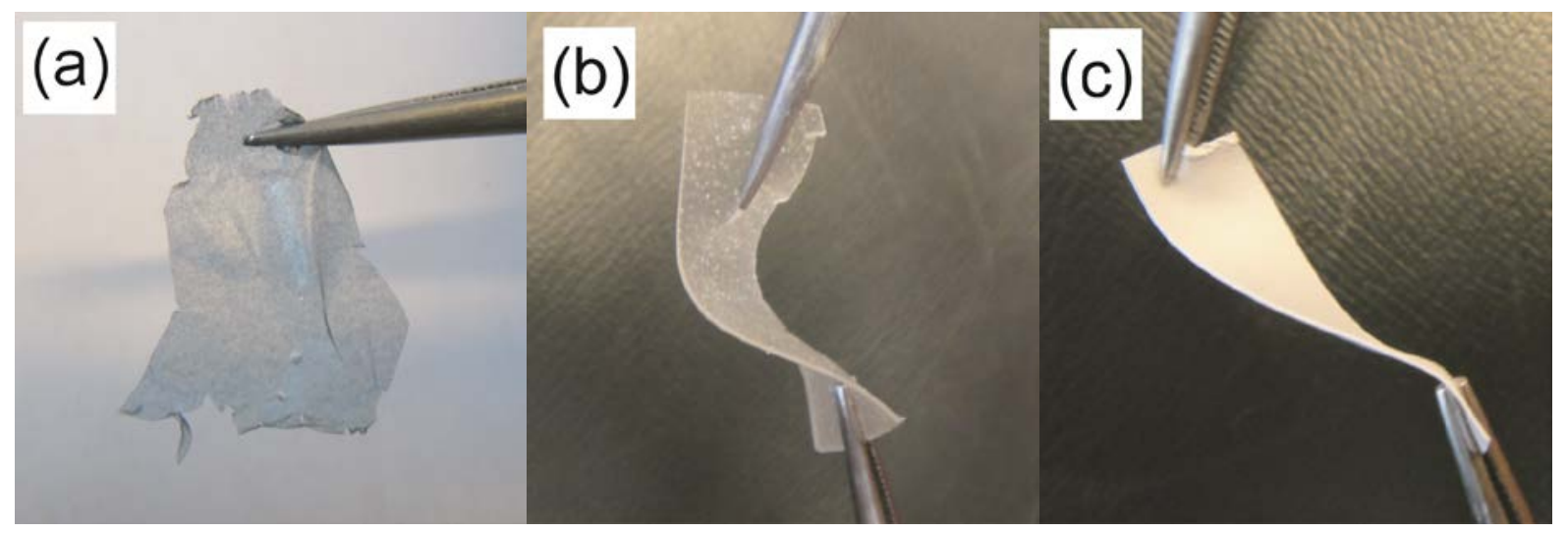

Fig. 1. Photographs showing the self-supporting films based on (a) NFC and PEDOT:PSS, (b) NFC and transparent polyelectrolyte, and (c) NFC and opaque polyelectrolyte. 
(a)

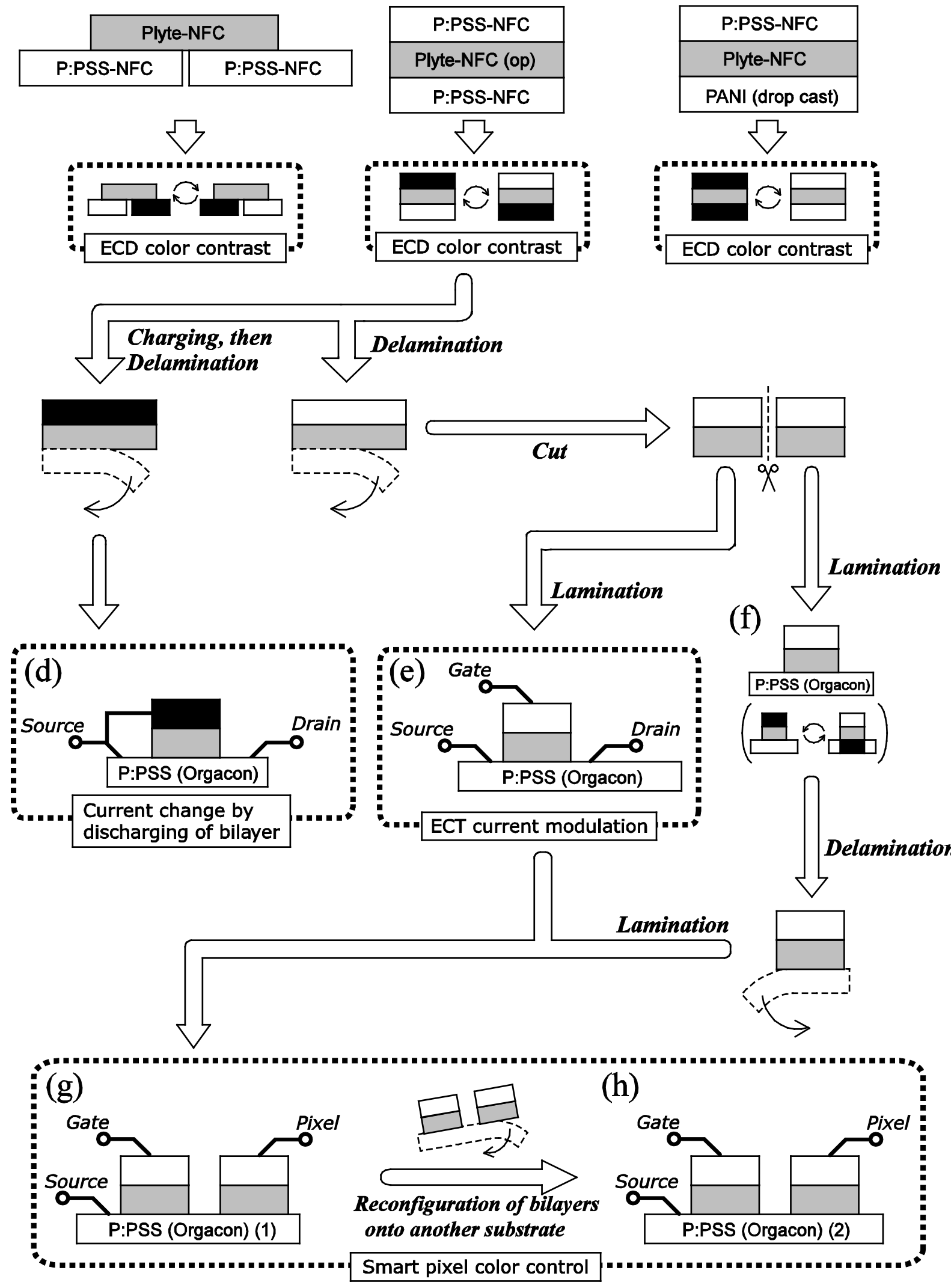

(c)

\begin{tabular}{|c|}
\hline P:PSS-NFC \\
\hline Plyte-NFC (op) \\
\hline P:PSS-NFC \\
\hline
\end{tabular}

(b)

.

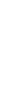


electrochromic electrodes of each ECD are ionically connected by either a transparent, (a) and (c), or an opaque, (b), electrolyte layer. (d) shows an ECT achieved by delamination and reconfiguration of a pre-charged (b) electrode that is applied onto an ECT channel patterned on top of a PET foil. The ECT in (e) is created after reconfiguring the (b) pixel electrode into an ECT gate electrode on top of a patterned ECT channel on a PET foil, while (f) proves that the ECD functionality can be maintained after reconfiguration. To fully demonstrate the concept, the color state of an ECD can be controlled by the conduction state of an ECT in an EC smart pixel device (g), which is obtained by delamination and reconfiguration of the (f) subcomponent onto the (e) device. Finally, this is further evidenced by simply duplicating the EC smart pixel device onto another substrate by utilizing the reconfiguration technique (h) and thereafter recording identical $I-V$ characteristics as compared to device (g). 


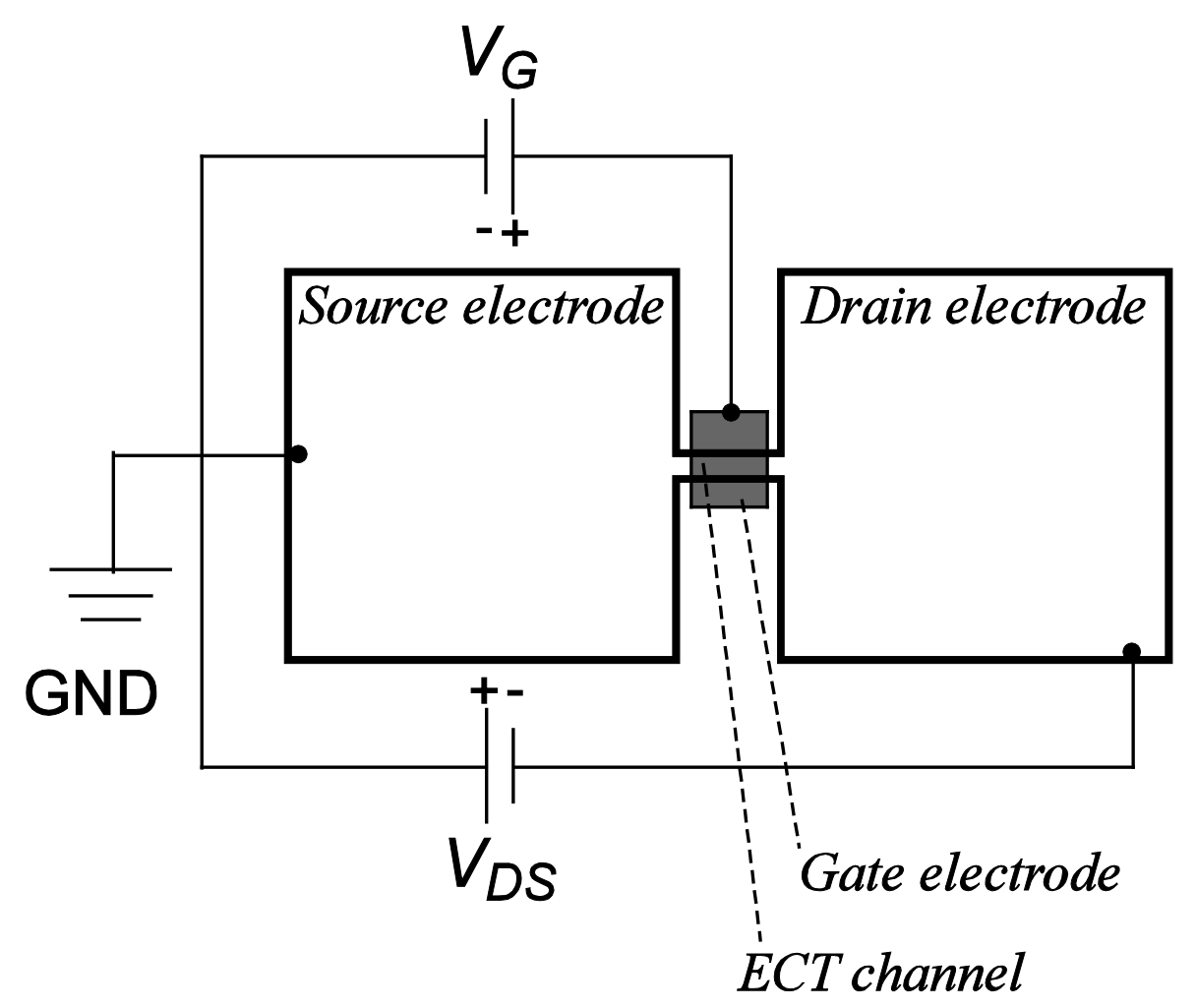

\section{$\square$ Patterned PEDOT:PSS surface on PET $\square$ Bilayer of P:PSS-NFC and Plyte-NFC}

Fig. 3. The top view of the ECT/EC smart pixel is illustrated. The drain and source electrodes are $20 \times$ $20 \mathrm{~mm}^{2}$ squares and they are connected by a narrow rectangle having an area of $0.4 \times 10 \mathrm{~mm}^{2}$. Approximately $3 \mathrm{~mm}$ of the length of the rectangle is covered by the electrolyte and the gate electrode bilayer, thereby forming the active area of the ECT channel, while an area of $2 \times 2 \mathrm{~mm}^{2}$ of the bilayer sheet was attached at the edge of the drain electrode such that the bilayer serves as the coloring pixel electrode and the drain electrode serves as the counter electrode of the EC smart pixel device. Note that the $V_{D S}$ is connected between the drain and source electrodes in the case of an ECT measurement, while the source and pixel electrodes are connected in the EC smart pixel measurement. 


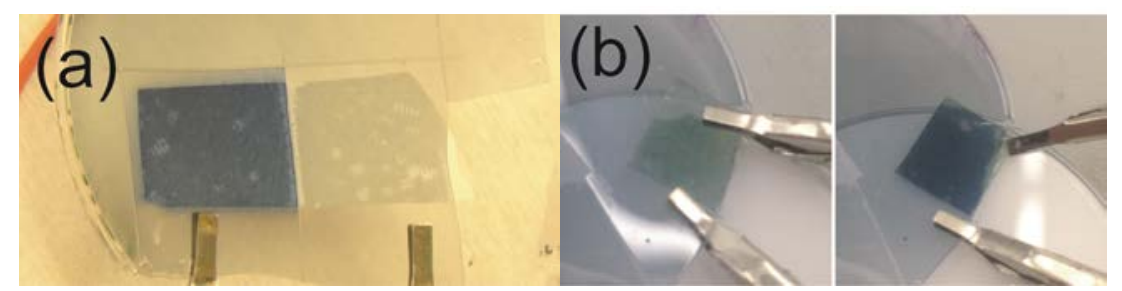

Fig. 4. Photographs showing two different display architectures that have been characterized: (a) lateral symmetric ECD based on two P:PSS-NFC electrodes bridged by Plyte-NFC, and (b) complementary vertical non-symmetric ECD consisting of Plyte-NFC sandwiched by one layer of P:PSS-NFC and a drop cast layer of PANI. No photograph is available for the vertical symmetric ECD based on opaque Plyte-NFC sandwiched by P:PSS-NFC on both sides.

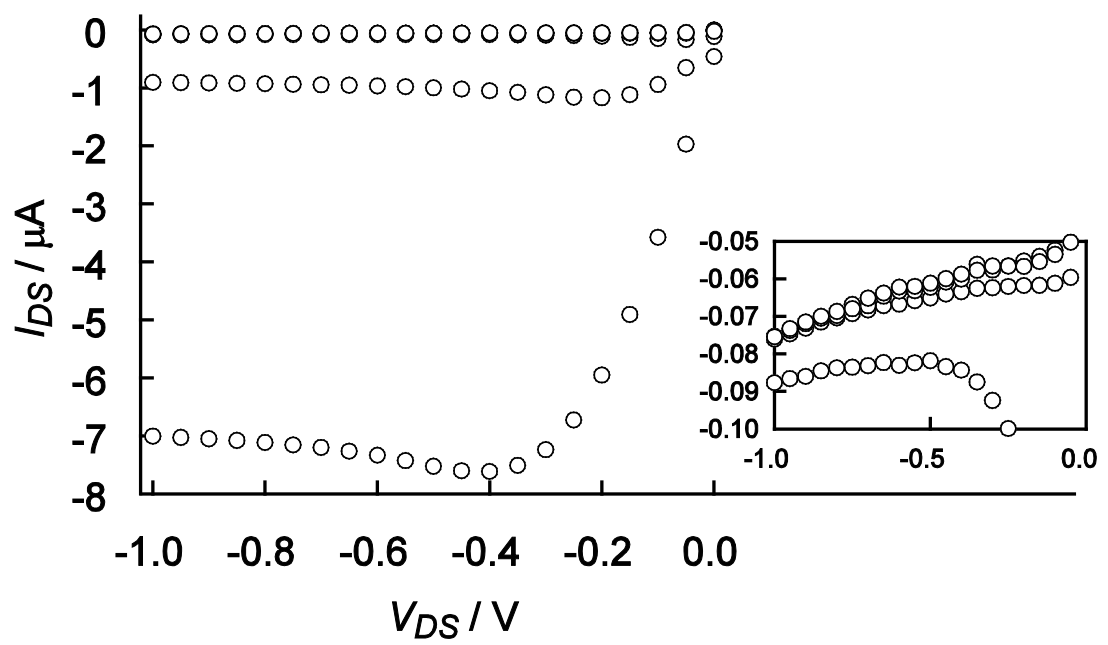

Fig. 5. $I-V$ characteristics of an ECT utilizing Orgacon as the transistor channel and the drain and source electrodes, Plyte-NFC as the electrolyte and P:PSS-NFC as the gate electrode. The inset graph indicates the off-current levels at elevated gate voltages.
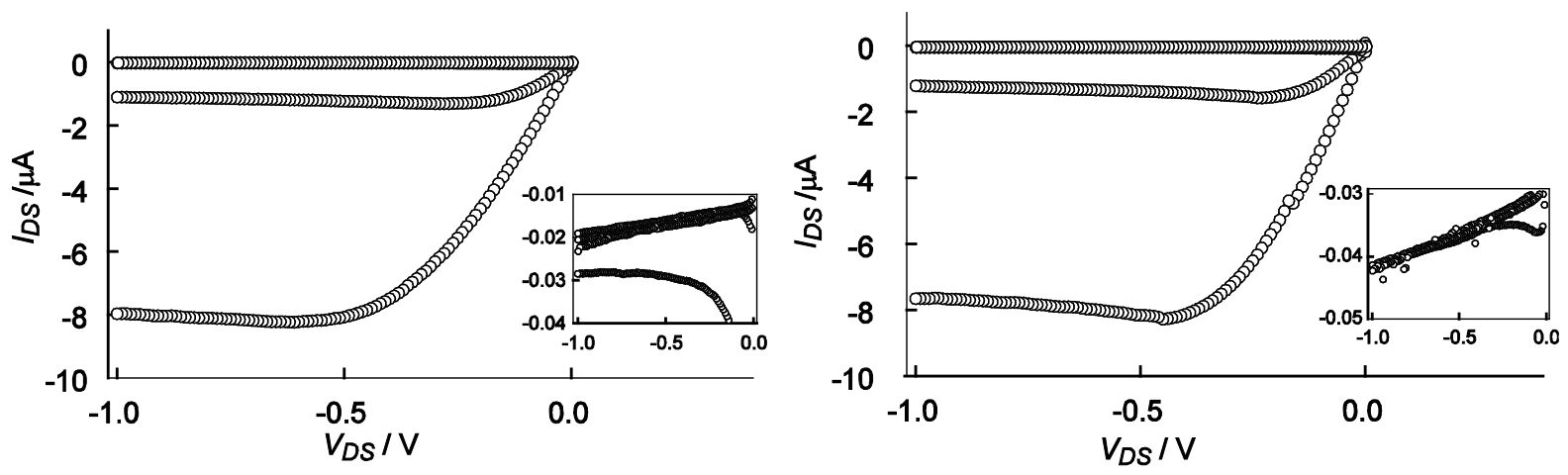

Fig. 6. An ECT showing identical $I-V$ characteristics 19 (left) and 31 (right) days after device manufacturing, respectively. The inset graphs indicate the off-current levels at elevated gate voltages. 


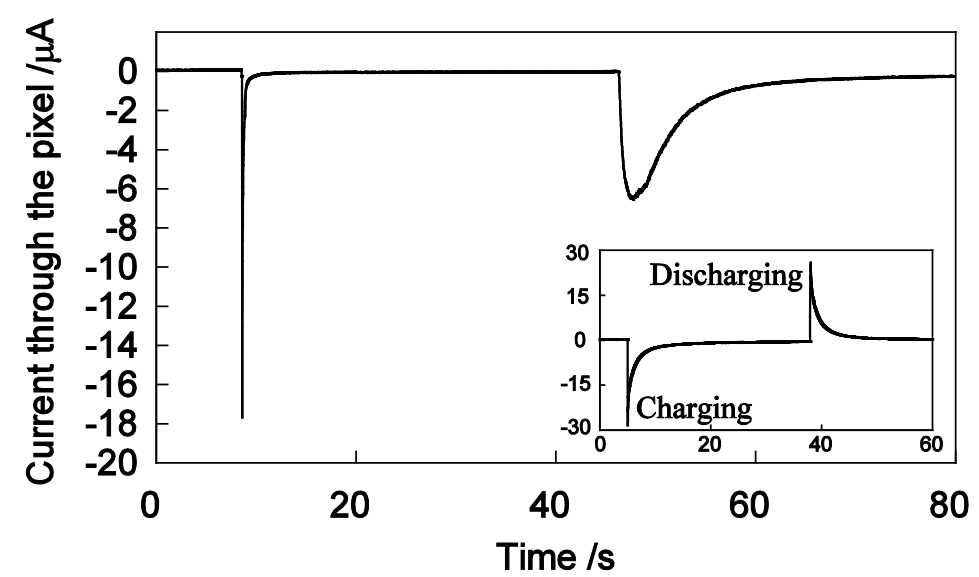

Fig. 7. The ECT shows that it can prevent ECD cross-talk. The first current peak at $t \sim 10 \mathrm{~s}$ corresponds to electrochemical reduction of the channel into its non-conducting state by applying $\mathrm{V}_{\mathrm{G}}$. $V_{D S}$ was applied at $t \sim 25 \mathrm{~s}$ but no current peak due to pixel coloration can be observed, which indicates that the off-state of the ECT is capable of keeping the ECD in its initial off-state. Finally, $V_{G}$ $=0 \mathrm{~V}$ is applied at $t \sim 45 \mathrm{~s}$, which brings the ECT channel to its on-state that, in turn, allows for pixel coloration by the constantly applied $V_{D S}$. The inset graph shows the charging and discharging behavior of an independent ECD.



Fig. 8. The ECT shows its capability to maintain the color state of the ECD. First, the pixel is switched to its colored state by $V_{D S}$, which is shown in the first current peak at $t \sim 3 \mathrm{~s}$. Subsequently $V_{G}$ is set to $1 \mathrm{~V}$ and the transistor channel switches off to its reduced state at $\mathrm{t} \sim 20 \mathrm{~s}$, which generates the second current peak. At $t \sim 32 \mathrm{~s} V_{D S}$ is set to $0 \mathrm{~V}$, which results in maintained color even though a small leakage current can be observed. When $V_{G}$ is set to $0 \mathrm{~V}$ at $t \sim 43 \mathrm{~s}$, the ECD is decolored by the discharging current indicated by the positive current peak. 


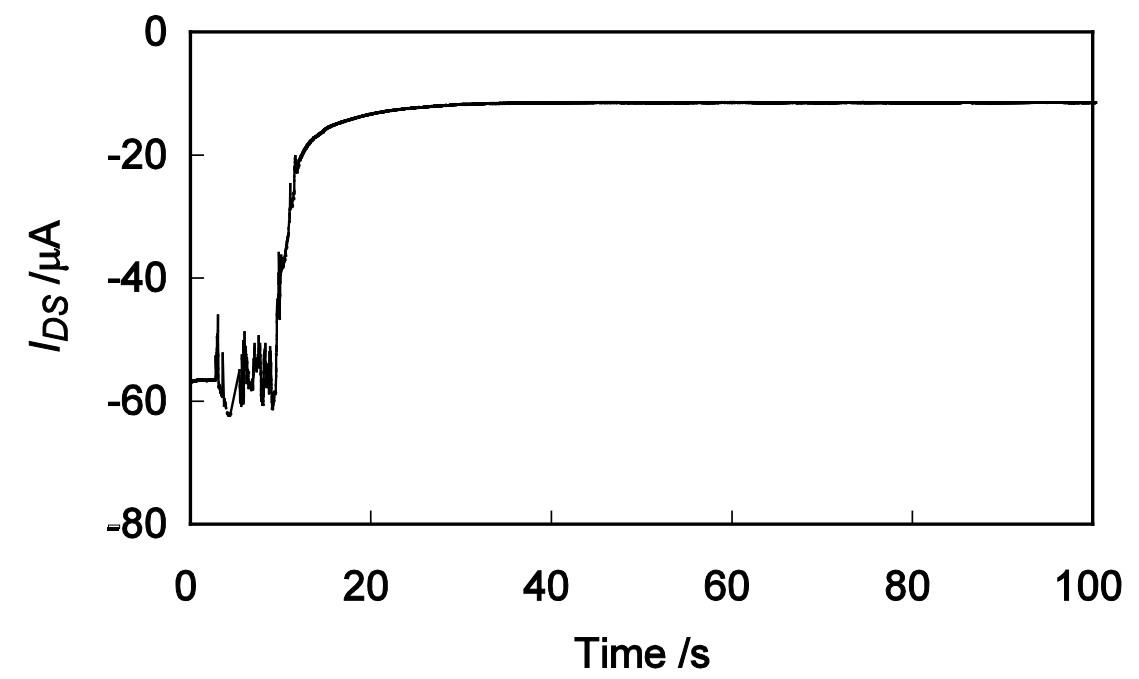

Fig. 9. The graph shows the current modulation of an ECT channel. The gate electrode was precharged adjacently to the ECT device, peeled off and reconfigured on top of the ECT channel. Before laminating the precharged gate electrode and its electrolyte layer, $I_{D S} \sim 55 \mu \mathrm{A}$ can be observed during the first few seconds of the measurement. Upon reconfiguration of the charged gate electrode, $I_{D S}$ decreases to $\sim 10 \mu \mathrm{A}$; far from the most optimized on/off-ratio in terms of transistor performance but yet it proves the concept of system integration, reconfiguration and energy transfer by using the peel and stick technique. 